\title{
MicroscopyAwards
}

\section{Microscopy Society of America Awards: 2020 Award Winners}

\author{
Edited by Bob Price ${ }^{1 \star}$ and Miaofang $\mathrm{Chi}^{2}$ \\ ${ }^{1}$ University of South Carolina \\ ${ }^{2}$ Oak Ridge National Laboratory \\ *Bob.Price@uscmed.sc.edu
}

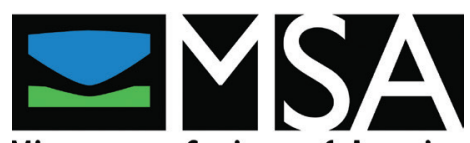

Microscopy Society of America
Each year, the Microscopy Society of America (MSA) provides several major awards for outstanding contributions to the fields of microscopy and microanalysis and for service to the Society. While recipients of these awards are listed under the tab Awards \& Scholarships - Society Awards | Microscopy Society of America on the MSA homepage, little information as to why awards were bestowed is provided. In this initial installment of what will become an annual contribution to Microsocopy Today, Miaofang Chi, Chair of the MSA Awards Committee, and I provide a perspective on the important contributions of the 2020 Award winners. The information presented here represents a short summary of information provided in the awardees' nomination packages. Guidelines, including deadlines for nominating individuals for these and other MSA awards, can be found by following the above link.

\section{Distinguished Scientist Awards}

Distinguished Scientist Awards annually recognize a preeminent senior scientist, from each of the biological and physical sciences, who has a long-standing record of achievement during his or her career in the field of microscopy or microanalysis.

\section{Physical Sciences Distinguished Scientist: David Seidman}

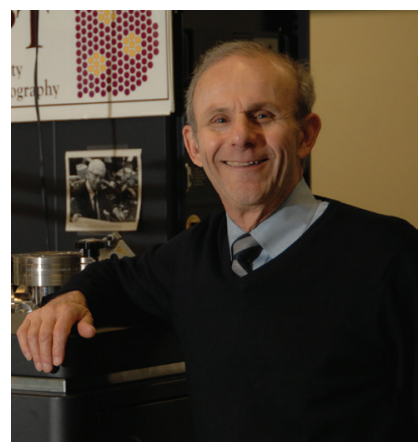

Broadly speaking, microscopy advances fall into two categories; those related to advancing microscope technology and those related to the applications of the technology. David Seidman is a rare individual who has advanced both. David is the embodiment of the physical materials scientist who embraces state-of-theart microscopy for the infor-

David Seidman, Walter P. Murphy Professor of Materials Science and Engineering, Northwestern University. mation it provides. His most outstanding contributions are primarily in the applications science. Yet, perhaps by necessity, he has actively pushed the development of microscopy science and technology, and especially atom probe field-ion microscopy (APFIM). Few of the great scientists in our field can fairly claim this combination of achievement.

As one of the early pioneers in the field of APFIM, Seidman established several basic capabilities of the technology. He established the first laboratory in the world dedicated to study quantitatively the fundamental properties of point defects in quenched or irradiated metals with FIM. To understand the basic physics of FIM, he initiated a series of experiments on the process of field-ionization, which involves the quantum mechanical tunneling of an imaging gas atom outermost electron into an FIM specimen. He used film-based movies of FIM images of tungsten specimens as they field evaporated to discern vacancy distributions in ion irradiation displacement cascades. In 1970, his three-dimensional reconstructions of the displacement cascades illuminated this basic phenomenon for the first time, and the work still stands as the only three-dimensional images of vacancies in materials nearly 50 years later. With approximately 500 publications and 20,000 citations of his work, Seidman exemplifies the title of Distinguished Scientist.

\section{Biological Sciences Distinguished Scientist: Xiaowei Zhuang}

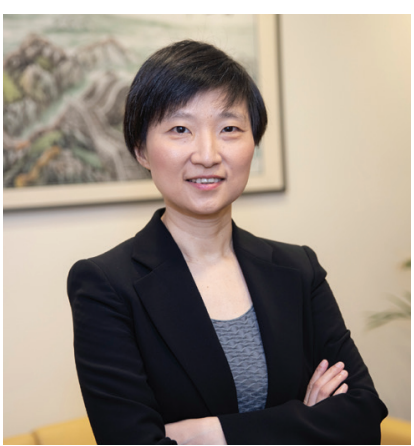

Xiaowei Zhuang, Howard Hughes Medical Institute Investigator and David B. Arnold Professor of Science, Harvard University. many subcellular structures. STORM overcomes the diffraction limit by exploiting Zhuang's discovery of photoswitchable dyes to separate spatially unresolvable molecules in time. In her original STORM paper, Zhuang demonstrated two-dimensional fluorescence imaging of biomolecular structures with $20 \mathrm{~nm}$ resolution, a drastic improvement over the diffraction limit. A major milestone in the super-resolution imaging field, Zhuang's 2006 paper has been cited approximately 5,500 times. 
More recently, Zhuang invented a first-in-kind single-cell transcriptome-imaging method-multiplexed error-robust fluorescence in situ hybridization (MERFISH)-which took molecular imaging to the genome scale and has revolutionized the field of single-cell genomics. Single-cell transcriptomic analyses are critical to understanding the molecular origin of cell behavior and tissue function. Yet, conventional sequencingbased single-cell transcriptomics approaches require dissociation of cells from tissues and extraction of RNAs from cells; therefore, the spatial information of RNAs and cells is lost. This loss of spatial information represents a major limitation, as the spatial organization of cells is critical to the development and function of tissues. Zhuang's MERFISH method has overcome this major challenge in the field and enabled in situ, spatially resolved, single-cell transcriptomics.

\section{Burton Medal}

The Burton Medal honors annually the distinguished contributions to the field of microscopy and microanalysis by a scientist who is less than 40 years of age on January 1st of the award year (that is, the awardee to be honored at M\&M 2020 cannot have been born earlier than 1980).

\section{Burton Medal: Brent Nannenga}

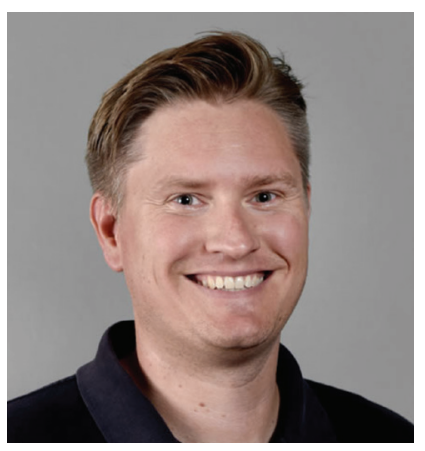

As a postdoctoral researcher in the laboratory of Tamir Gonen at the Howard Hughes Medical Institute's Janelia Research Campus, Dr. Nanenga's research focused on cryo-EM and the development of a new method in electron diffraction called microcrystal electron diffraction, or MicroED. Since the initial MicroED publication Brent Nannenga, Assistant Professor in 2013, it has been used to of Chemical Engineering, School for solve numerous high-resoluEngineering of Matter, Transport and tion structures from very thin Energy, Arizona State University.

as a postdoctoral researcher, Brent was involved in the publicathe publication of 7 papers on MicroED, which were in journals such as Nature, Nature Methods, and eLife. Initially developed using protein microcrystals, MicroED has since been extended to the analysis of peptides and small organic molecules, with many of these being novel structures that were unable to be determined using other structural methods. There has been great interest in this technique from the microscopy and structural biology communities, because growing large crystals can be extremely difficult, and new microcrystallography techniques are in high demand.

Since starting his own research group at Arizona State University, Brent has continued to focus on the development and application of electron microscopy methods. One key area of research in his group is extending MicroED sample handling and data collection procedures to microcrystals, which are grown in the viscous lipidic cubic phase, specifically G-protein coupled receptors (GPCRs), which are an important class of membrane proteins that represent approximately $50 \%$ of all drug targets. In addition to his work on MicroED, Brent is studying the molecular interactions of biological molecules with inorganic materials, using both crystallography and single-particle cryo-EM.

\section{Burton Medal: James LeBeau}

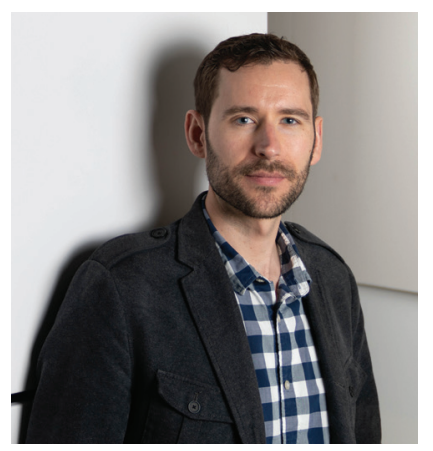

James LeBeau's research is focused on developing fundamental understanding of material properties through imaging and spectroscopy, and he has achieved this through the development of new techniques for data acquisition and analysis. His research has transformed the STEM field, expanding the scope of quantitative electron micros-

James LeBeau, Associate Professor, Department of Materials Science \& Engineering, Massachusetts Institute of Technology. copy in a way that enhances its applications to real-world problems. The techniques Jim has developed and advanced are being adopted widely across the microscopy and materials sciences communities, and they enable new insight into the atomic structure of materials and how structure translates into unique material functionality. Jim's contributions can be grouped into two classes, although these are, of course, interconnected: developing a suite of new methods in STEM acquisition and analysis and applying these methods to key materials problems.

The development of these methods was driven by Jim's interest in solving specific materials problems that could not be addressed using conventional STEM imaging, even at high resolution. The techniques are especially useful in understanding material properties that are influenced by local structure, for example, when local unit cell crystallography has lower symmetry than the averaged structure, or at material surfaces and interfaces. In such cases, the ability to perform quantitative STEM analysis using these innovative techniques opens the doorway to understanding nanoscale interactions and the influence of atomic constituents on a range of structurally driven functional properties. The methods are powerful tools for the analysis of material crystallography, complementing diffraction-based pair distribution function techniques that otherwise require access to national beamline facilities, while also having the benefit of spatial resolution and correlation with local chemistry measurements.

\section{Albert Crewe Award}

The Albert Crewe Award was initiated to recognize annually the distinguished contributions to the field of microscopy and microanalysis in the physical sciences of an early career scientist, of not more than 6 years' standing since doctoral graduation, for research performed during this period.

\section{Crewe Award: Andrew Yankovich}

Based on his groundbreaking research, Andy Yankovich is widely recognized as a leading young scientist in the materials 


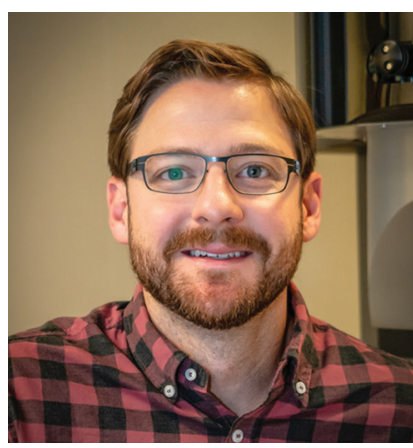

Andrew Yankovich, Research Specialist, Department of Physics, Chalmers University of Technology, Gothenburg, Sweden.

statistical treatment of experimental data give him the tools to create new approaches to solve difficult problems. His creativity, curiosity, independence, and enthusiasm continually drive him into new areas of physics. His research findings have continued to receive highly positive response from the scientific community and have facilitated the work of many others. His exceptional skills as a materials-oriented electron microscopist, his demonstrated commitment to collaborative research, and his leadership capabilities indicate that he has the potential to continue making leading contributions well into the distant future.

\section{Hildegard H. Crowley Award for Outstanding Technologist in the Biological Sciences}

This award annually honors a technologist from the biological sciences who has made significant contributions, such as the development of new techniques that have contributed to the advancement of microscopy and microanalysis. A technologist is defined as an individual whose primary role is in microscopy and microanalysis tool development or service. Established principal investigators/project managers are not eligible for this award.

\section{Crowley Award: Joseph Mowery}

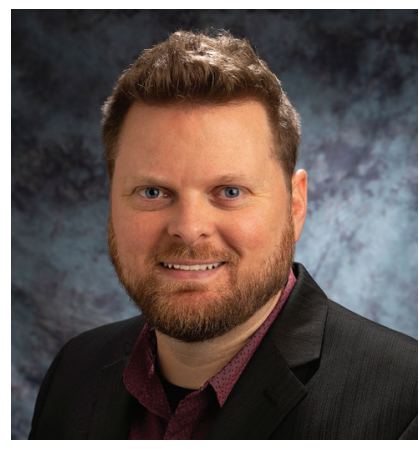

Joe has a diversified training background and has made significant contributions to the morphological characterization of a protozoan parasite, Sarcocystis, an emerging pathogen in countries where people consume meat from wild animals and have lower standards of food safety. Joe was also involved in the morphological characterizations of mites, Joseph Mowery, Biologist, Electron \& Confocal Microscopy Unit, U.S. Department of Agriculture, Agricultural Research Service, Beltsville, MD.

tron \& Crops. In his work at the Elecment of Agriculture, Agricultural Research Service, Beltsville, $\mathrm{MD}$, Joe has often encountered unique and rare specimens that necessitated he apply innovative measures to optimize preservation and EM processing. For instance, Joe developed a microwave embedding protocol specifically for the preservation and resin embedding of plant and woody material. He was invited to present this work at the Microscopy \& Microanalysis meeting in Baltimore in 2018. Most recently, Joe played a significant role in research that demonstrated that Varroa mites feed primarily on honeybee fat body tissue, not hemolymph as previously thought. This work was published in the Proceedings of the National Academy of Sciences in January 2019. Joe's clever design of a feeding arena for mites and of a method for immobilizing feeding mites on the honeybee enabled the precise localization and characterization of the feeding site and target tissue. This work overturned the conventional belief that Varroa mites feed on the hemolymph of the honeybees and may have significant implications in the future development of management schemes for this devastating honeybee parasite.

\section{Chuck Fiori Award for Outstanding Technologist in the Physical Sciences}

This award annually honors a technologist from the physical sciences who has made significant contributions, such as the development of new techniques that have contributed to the advancement of microscopy and microanalysis. A technologist is defined as an individual whose primary role is in microscopy and microanalysis tool development or service. Established principal investigators/project managers are not eligible for this award.

\section{Fiori Award: Lijun Wu}

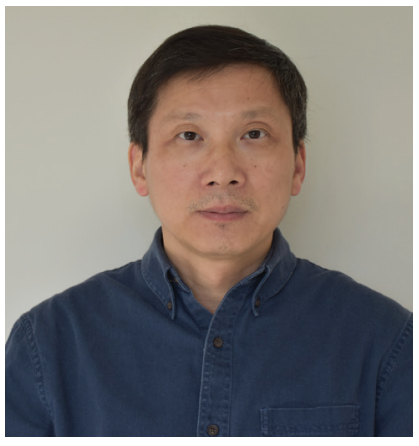

Lijun joined Brookhaven National Laboratory (BNL) in 1996 as a visiting researcher, with a Master's degree in Materials Science, and was soon promoted to Technical Staff in the Applied Science Department at the BNL. Based on the excellent research results that he acquired at $\mathrm{BNL}$, he received his $\mathrm{PhD}$ in 2002. Lijun has been critical to the success of research at BNL,

Lijun Wu, Engineer I, CMPMS Division, Brookhaven National Laboratory.

both at the electron microscopy user facility in the Center for

Functional Nanomaterials and in the DOE/BES core-research programs. Lijun's exceptional contributions stem from his knowledge of electron crystallography and computer programming. The computer codes he has developed have been widely used in materials science research, allowing better understanding of the relationships between structure and properties. As an example, he and his colleagues developed the unique quantitative diffraction technique "parallel recording of dark field image (PARODI)" to accurately measure structural factors of both low- and high-order reflections. The low-order structure factors are used to map valence electron distribution in functional materials, while the high-order ones reveal anharmonic thermal motion of atoms.

With his proficiency in computer programming, Lijun has developed advanced computer programs for image processing, 3D structural modeling, HRTEM/STEM image simulation 
based on a multi-slice method with frozen phonon approximation, dynamic electron diffraction calculations based on Bloch wave method with structure factors, and atomic position refinement. With a multi-slice algorithm he did the first atomic-resolution SEM simulation and solved the contrast reversal puzzle in annular dark-field (ADF) images in STEM.

\section{Morton D. Maser Distinguished Service Award}

This award recognizes outstanding volunteer service to the MSA as exemplified by Mort Maser, who served the Society for many years with great dedication. This award is made annually to honor an MSA member who has provided significant volunteer service to the Society over a sustained period of time.

\section{Maser Award: John Shields}

John Shields has been a dedicated MSA volunteer for more than two decades. His first roles were on the Education Committee and at the M\&M Program Production Meetings, where the M\&M annual meeting is planned. However, perhaps

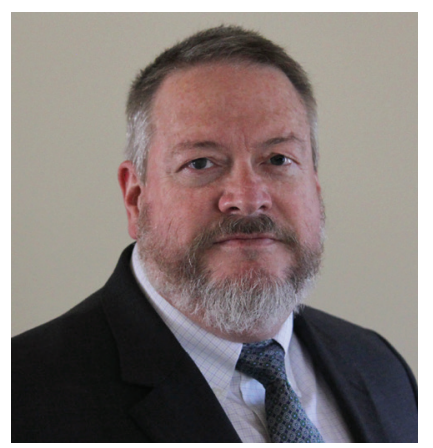

John Shields, Managing Director, Georgia Electron Microscopy and Academic Professional, Department of Cellular Biology, University of Georgia. of Chair of the Archives Committee in the near future. John's willingness to volunteer his time and expertise exemplifies dedication to the MSA.

\section{MSA Fellows}

The designation MSA Fellow is intended to recognize those who have been conferred the Society's Distinguished Scientist Awards, as well as senior distinguished members of the Society who have been a member of the Society for at least 10 years and have made significant contributions to the advancement of the field of microscopy and microanalysis through a combination of scientific achievement, service to the scientific community, and the Society itself. Election to MSA Fellow is highly selective and represents a broad cross section of the MSA membership. The number of MSA members elected to Fellowship each year is restricted to no more than $0.5 \%$ of the total MSA membership. For each Fellow, the nominating statement supporting their award is italicized followed by a short summary of their service to the MSA or microscopy research community.

\section{MSA Fellows with Nominating Statement}

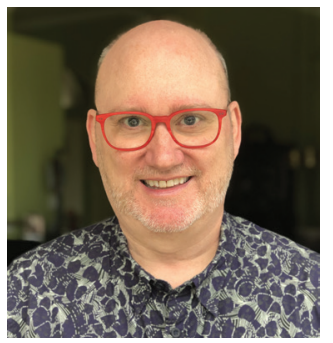

Ian M. Anderson, Summerfield Research Institute.

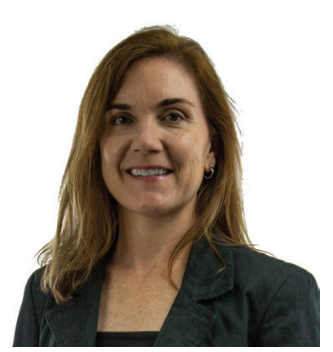

Elizabeth C. Dickey, Department Head and Teddy and Wilton Hawkins Distinguished Professor, Materials Science and Engineering, Carnegie Mellon University.
Ian M. Anderson: For his long-term and tireless efforts at MSA, including the initiation of the Student Council, a path for the future of the Society. For his scientific contributions to HREM, $A E M$, spectroscopy, elemental mapping, and atom probe analysis. Ian also served as the M\&M 2004 Program Chair and as a long-standing MSA Council member, including six years as Secretary and President in 2017.

Elizabeth C. Dickey: For her outstanding microscopy contributions to the understanding of oxide nanostructures and their relevance to diverse fields including catalysis, fuel-cells and structural mechanics. Beth's work has been cited more than 14,000 times, with several papers having more than 500 citations each. She also has a long record of service to MSA, including as a Director, and is now the Editorin-Chief of Elements, which is a new digital publication of MSA.

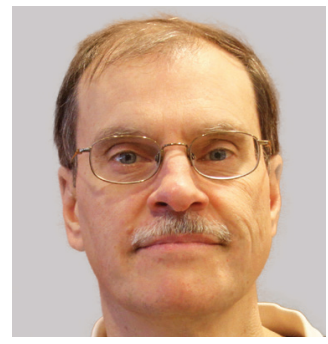

J. Bernard Heymann, Staff Scientist, Laboratory of Structural Biology Research, National Institute of Arthritis and Musculoskeletal and Skin Diseases, National Institutes of Health.

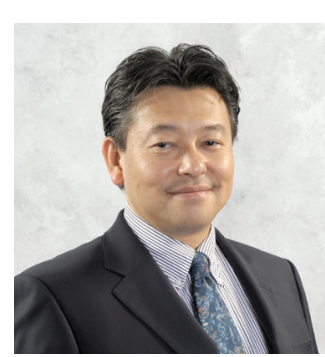

Masashi Watanabe, Associate Professor, Materials Science and Engineering, Lehigh University and Associate Director, Lehigh Materials Characterization Facility.
J. Bernard Heymann: An internationally renowned specialist in software for biological electron microscopy. He has created, applied, and widely supports Bsoft, a powerful package for image reconstruction and electron tomography. His extensive work in biological- and cryo- electron microscopy has led to numerous advances in our understanding of single-particle, protein, and virus structure.

Masashi Watanabe: For outstanding contributions to quantitative STEMXEDS microanalysis through his development of the zeta factor technique, and to the development of atomic-level microanalysis techniques for analytical electron microscopy. Masashi has been a long-time contributor to $M \& M$ meetings, having chaired numerous sessions, and is also an Editor for Microscopy and Microanalysis. 